\title{
Efficacy and safety of combination therapy with latanoprost after a change in therapeutic regimen from timolol to brinzolamide in Japanese adult patients with primary open-angle glaucoma and ocular hypertension: open, non-randomized I2-week study
}

\author{
Shusaku Ishikawa' \\ Yoshimi Nakamura' \\ Yuko Nakamura' \\ Hiroshi Sakai ${ }^{\prime}$ \\ Shoichi Sawaguchi' \\ Kazuo Terashima ${ }^{2}$ \\ Makoto Kanno ${ }^{2}$ \\ Hidetoshi Yamashita ${ }^{2}$ \\ 'Department of Ophthalmology, \\ University of the Ryukyus Faculty \\ of Medicine, Okinawa, Japan; \\ ${ }^{2}$ Department of Ophthalmology \\ and Visual Science, Yamagata \\ University Faculty of Medicine, \\ Yamagata, Japan
}

Purpose: To compare the efficacy of brinzolamide in Japanese patients with primary open-angle glaucoma (POAG) or ocular hypertension $(\mathrm{OH})$ after a change from timolol in combination therapy with latanoprost.

Methods: A 12-week, prospective, open-label, comparative study was performed in 20 patients [11 males and 9 females, mean age of $64.5 \pm 11.0$ (SD)y] with POAG or OH treated with both latanoprost once daily and timolol $0.5 \%$ twice daily. During the study brinzolamide was substituted for timolol. Intraocular pressure (IOP) was measured at baseline, 4, 8, and 12 weeks. Blood pressure (BP), pulse rate (PR), and adverse events were also recorded.

Results: IOPs at baseline, 4,8 , and 12 weeks were $18.6 \pm 2.1 \mathrm{mmHg}, 17.8 \pm 2.6 \mathrm{mmHg}$, $17.4 \pm 2.5 \mathrm{mmHg}$, and $17.3 \pm 3.5 \mathrm{mmHg}$, respectively. IOP reduction at 4 and 8 weeks was statistically significant $(\mathrm{p}<0.05)$. The PR was significantly increased at 12 weeks $(\mathrm{p}<0.01)$, but BP was not significantly affected. Four ocular adverse events were noted, but all were mild and transient.

Conclusions: Substituting brinzolamide $1 \%$ for timolol $0.5 \%$ in combination therapy with latanoprost $0.005 \%$ demonstrated significant IOP reduction with improvement in PR with $\mathrm{POAG}$ or $\mathrm{OH}$. Combination therapy using latanoprost and brinzolamide may be recommended for better IOP control with fewer systemic adverse events.

Keywords: open-angle glaucoma, brinzolamide/latanprost combination therapy, timolol/ latanoprost combination therapy, intraocular pressure

\section{Introduction}

In recent years, significant advances have been achieved in the development of topical glaucoma medications (Camras et al 1996; Silver 1998; Brubaker et al 2000), and options for combination therapy have increased. Because a significant proportion of glaucoma patients eventually need more than one agent for adequate control of intraocular pressure (IOP) (Kobelt-Nguyen et al 1998), it is important to reduce adverse ocular or systemic events in those treated with combination therapy. Using prostaglandin analogs as first-line treatment has given rise to numerous studies in search for the most effective and best-tolerated combination, from both a local and a systemic perspective. IOP reduction rates with various agents have been reported as 
follows: timolol $0.5 \%$ monotherapy, approximately $23.4 \%$ (Schenker et al 1999); topical carbonic anhydrase inhibitors (CAIs) such as brinzolamide, 13.2\%-16.7\% (Sall 2000); and dorzolamide, 19.4\% (Arici et al 1998). Meta-analysis of the IOP-lowering effects of several glaucoma medications demonstrated similar results (van der Valk 2005), with IOP reduction rates being highest with beta-blockers. The combination of a prostaglandin analog plus a betablocker has been extensively studied and is probably the most commonly used combination (Alm et al 1995; Larsson 2001; Higginbotham et al 2002). It is noteworthy that the addition of a topical beta-blocker to latanoprost provides a slight additional IOP reduction (Bucci 1999; O'Connor et al 2002). The efficacy of combination therapy using a topical CAI and latanoprost has not been studied to the same extent as that of latanoprost in combination with a beta-blocker; however, dorzolamide and brinzolamide have been reported to lower IOP at least as effectively as a beta-blocker when added to latanoprost (O'Connor et al 2002; Shoji 2005). This option of latanoprost and topical CAI combination therapy may confer advantages for glaucoma patients as both drugs present an excellent systemic safety profile.

Brinzolamide ( $1 \%$ ophthalmic solution) is a topical CAI that, like dorzolamide, is known to lower IOP by inhibiting the production of aqueous humor (Silver 1998; Schenker et al 1999; Brubaker et al 2000; DeSantis 2000; March and Ochsner 2000; Michaud and Friren 2001). The IOP-lowering effect of brinzolamide $1.0 \%$ is reported to be equivalent to that of dorzolamide $2 \%$, both as monotherapy (Barnebey and Kwok 2000; Sall 2000) and in combination therapy with timolol (Michaud and Friren 2001). Furthermore, when brinzolamide is substituted for dorzolamide, IOP is further decreased (Barnebey and Kwok 2000) or is equal to IOP before substitution (Kobayashi et al 2004; Kubota et al 2004). In evaluations of ocular adverse events, dorzolamide has reportedly caused more intense ocular irritation than brinzolamide (Barnebey and Kwok 2000; Sall 2000; Silver 2000; Michaud and Friren 2001; Seong et al 2001; Kobayashi et al 2004; Stewart et al 2004; Tsukamoto et al 2005). The incidence of ocular discomfort or blurred vision for brinzolamide is significantly higher than that of dorzolamide in one study (Silver 2000), but not statistically significant in other studies (Sall 2000; Michaud and Friren 2001; Stewart et al 2004; Tsukamoto et al 2005). Thus brinzolamide 1\% twice daily was selected as the topical CAI in this study.

The objective of this study was to evaluate the effects of a change in therapeutic regimen from combination therapy with timolol/latanoprost to combination therapy with brinzolamide/latanoprost in terms of: IOP, the cardiovascular functions of blood pressure (BP) and pulse rate (PR), and ocular adverse events.

\section{Patients and methods}

A 12-week, open-label, non-randomized, prospective, comparative study was conducted in Japanese patients with primary open-angle glaucoma (POAG) (17 patients) or ocular hypertension $(\mathrm{OH})$ (3 patients) at Ryukyu University Hospital and Yamagata University Hospital. POAG was defined as glaucomatous optic neuropathy with repeatable visual field defects, and open angle. $\mathrm{OH}$ was defined as a normal visual field and normal optic disc appearance. Enrolled patients had been under treatment with both latanoprost (once daily, at $8 \mathrm{PM}$ ) and timolol $0.5 \%$ (twice a day, at 8 AM and $8 \mathrm{PM}$ ) for 3 months or longer and had not used any topical CAI prior to this study.

Inclusion criteria for the study were: (1) patients with primary open-angle glaucoma or ocular hypertension, (2) patients who had been treated with both latanoprost $0.005 \%$ once daily and timolol $0.5 \%$ twice daily for more than 3 months, (3) patients with IOP on latanoprost and timolol treatment $\geq 16 \mathrm{mmHg}$, (4) patients aged 20 years or over. The study included both males and females and inpatients and outpatients.

The following patients were excluded from the study: (1) patients with a history of chronic or recurrent inflammatory eye disease, ocular trauma, or ocular infection within the past 3 months, (2) those with corneal abnormalities preventing reliable applanation tonometry, (3) those who had had intraocular surgery, (4) those who had had laser surgery within the past 3 months, (5) those with severe, unstable, or uncontrolled cardiovascular or pulmonary disease that would preclude use of an ophthalmic beta-blocker, (6) those currently using any ophthalmic, dermatologic, or systemic corticosteroid or oral CAIs, (7) those with a history of severe hypersensitivity to oral CAIs, sulfonamide drugs, or any components of these medications, (8) those with dementia, and (9) those whom the investigator determined ineligible to participate in this study. Because enrolled patients were eligible for latanoprost and timolol combination therapy, those with hypersensitivity or allergy to either of these agents were excluded prior to the study.

The study protocol followed the guidelines of the Declaration of Helsinki. All patients were given a thorough explanation of the aims, protocol, and all procedures of the study before enrollment, and informed consent was then obtained. 
Binzolamide $1 \%$ twice daily (at $8 \mathrm{AM}$ and $8 \mathrm{PM}$ ) was given to each patient in place of timolol $0.5 \%$ twice daily to compare the efficacy and side effects from this change in therapeutic regimen. There was no wash-out period between the two treatment regimens. The prescription of brinzolamide $1 \%$ twice a day is common practice and is based on the label approved by the government in Japan.

IOP was analyzed on 1 eye per patient. If both eyes were eligible for inclusion, the eye with higher baseline IOP was selected as the study eye. When IOP was the same in both eyes, the right eye was selected. IOP was measured at the baseline visit and at 4, 8, and 12 weeks after enrollment using Goldmann applanation tonometry. The time of day at which IOP was measured during the study was the same as that at baseline. IOP measured immediately prior to substituting brinzolamide $1 \%$ was considered as the baseline. Resting BP (systolic and diastolic) and PR were also measured at the baseline and at 12 weeks. All patients underwent ophthalmic examinations at each visit during the follow-up period. Subjective ocular discomfort (mild irritation, blurred vision, and conjunctival hyperemia) and subjective ocular adverse events (stinging/intense irritation) after instillation of eye drops were investigated by patient interview. During clinic visits patients were asked whether they had a sense of ocular discomfort at the time of instillation and how long the ocular discomfort persisted after instillation. If a patient complained of any sense of irritation and/or blurred vision at least once during the follow-up period, the patient was regarded as having ocular discomfort.

IOP, BP, and PR values are shown as mean \pm standard deviation. The Wilcoxon signed rank test was used for statistical analysis, and the difference was considered significant when the $\mathrm{p}$ value was less than 0.05 .

\section{Results}

Of the 20 patients ( 11 males and 9 females) enrolled, no patients dropped out during the course of the study, and therefore 20 patients were accepted for analysis of IOP and local and systemic adverse events. Table 1 shows the

Table I Patients

\begin{tabular}{ll}
\hline Glaucoma diagnosis & \\
Primary open angle glaucoma & 17 eyes \\
Ocular hypertension & 3 eyes \\
Female : Male & $9: 11$ \\
Age (Mean \pm standard deviation) & $64.5 \pm 11.0$ years \\
\hline
\end{tabular}

demograhpic characteristics of the patients. Briefly, the mean age of the patients was $64.5 \pm 11.0$ (mean \pm standard deviation) years old. Seventeen patients (17 eyes) had POAG, and 3 patients ( 3 eyes) had OH. All had been treated with a combination of latanoprost $0.005 \%$ and timolol $0.5 \%$ for more than 3 months. Figure 1 shows the course of IOP at baseline and after the regimen change. Mean IOP at baseline, 4, 8, and 12 weeks after enrollment were $18.6 \pm 2.1 \mathrm{mmHg}, 17.8 \pm 2.6 \mathrm{mmHg}$, $17.4 \pm 2.5 \mathrm{mmHg}$, and $17.3 \pm 3.5 \mathrm{mmHg}$, respectively. Significant reductions in mean IOP were observed at the 4-week and 8-week time points $(\mathrm{p}<0.05)$ (Figure 2). Mean IOP reduction from baseline was $0.8 \mathrm{mmHg}(4.3 \%$ reduction) after 4 weeks, $1.2 \mathrm{mmHg}(6.5 \%$ reduction $)$ after 8 weeks, and $1.3 \mathrm{mmHg}$ ( $7.0 \%$ reduction) after 12 weeks.

Mean systolic and diastolic BPs were $139 \pm 20 \mathrm{mmHg}$ and $80 \pm 12 \mathrm{mmHg}$ at baseline, and $135 \pm 24 \mathrm{mmHg}$ and $81 \pm 13 \mathrm{mmHg}$ at the 12 -week point. Thus the substitution of brinzolamide had little effect on systolic and diastolic BP $(p>0.05)$ (Figure 3).

In contrast, mean PR was $68 \pm 9 \mathrm{bpm}$ at baseline, with a significant increase to $78 \pm 13 \mathrm{bpm}$ at the 12 -week point $(\mathrm{p}<0.01)$ (Figure 4). Visual field defect and optic disc appearance did not change throughout the study period.

No patient reported the adverse event of intense ocular irritation. After therapeutic substitution, ocular adverse events were observed in 4 cases ( 2 blurred vision, 1 mild irritation, and 1 mild hyperemia) (Table 2). All were mild and transient and disappeared without any treatment. Systemic adverse events were not observed.

\section{Discussion}

In this study, we compared the efficacy and safety of brinzolamide $1 \%$ twice daily as a second-line medication

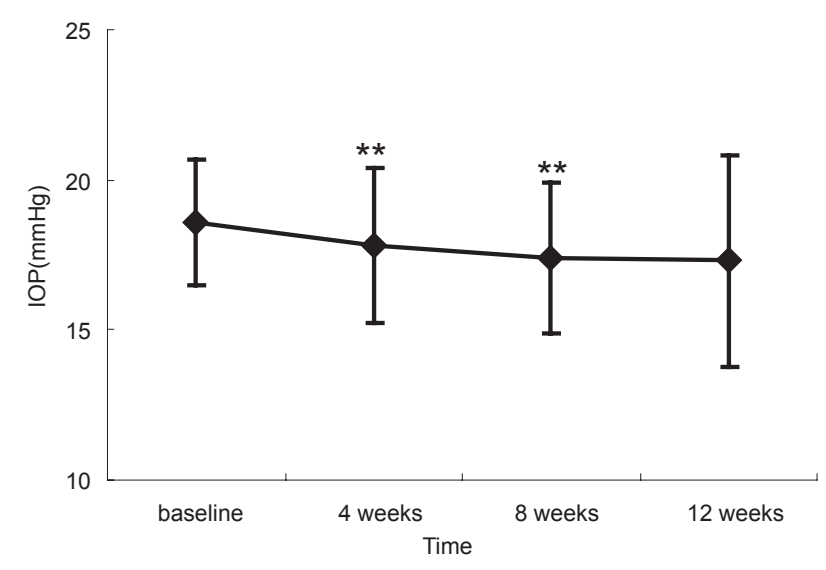

Figure I Change in IOP after regimen change. ( ${ }^{* *} \mathrm{p}<0.05$, Wilcoxon signed rank test). 


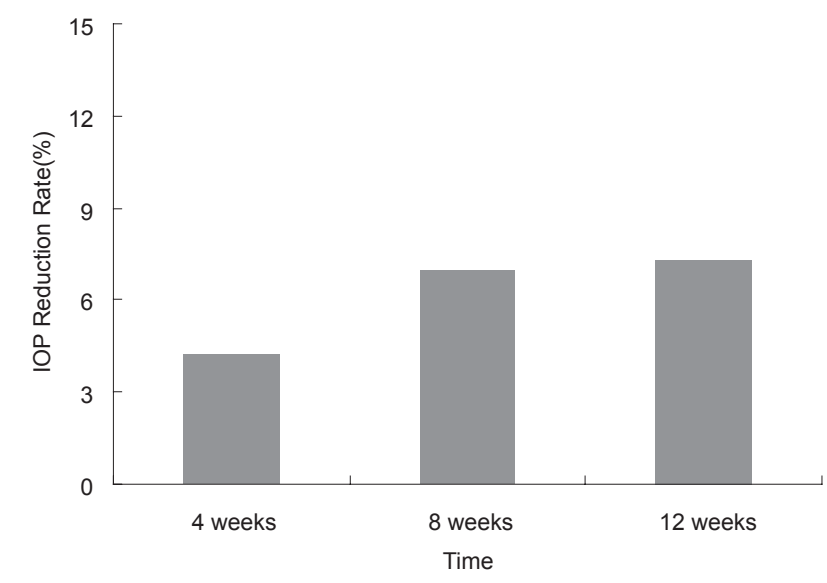

Figure 2 Change in IOP reduction rate after regimen change. Average IOP reduction rate 12 weeks after regimen change was $7.3 \%$.

when substituted for timolol $0.5 \%$ in combination therapy with latanoprost in Japanese patients with POAG or $\mathrm{OH}$. We concluded that substitution of brinzolamide for timolol significantly lowered IOP with significant improvement of PR without systemic or serious local adverse reactions.

\section{Efficacy}

Two recently developed topical CAIs, dorzolamide and brinzolamide, are now usually used as an adjunctive therapy to other anti-glaucoma eye drops. The IOP-lowering effect of brinzolamide $1 \%$ (twice daily) was equivalent to dorzolamide $2 \%$ (3 times daily) when added to timolol $0.5 \%$ (Michaud and Friren 2001). The effect of topical CAIs as a

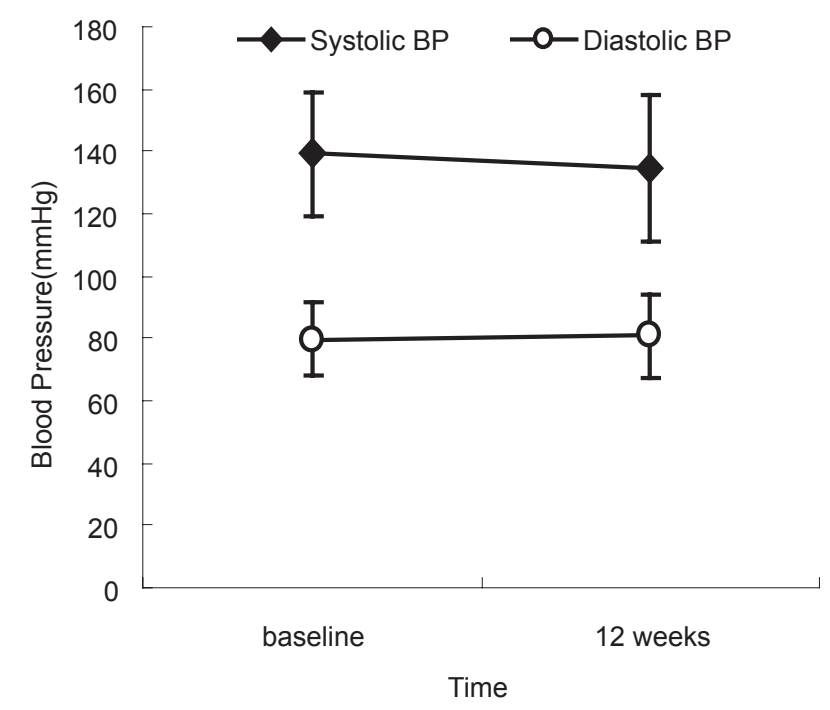

Figure 3 Change in blood pressure after regimen change. No significant differences were seen in blood pressure ( $p>0.05$, Wilcoxon signed rank test).

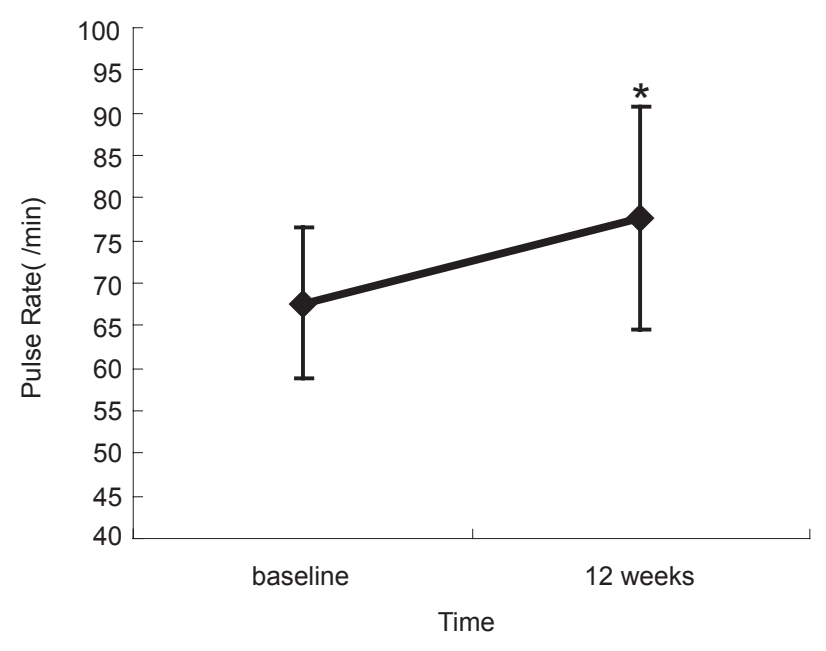

Figure $4 \mathrm{Change}$ in pulse rate after regimen change. The pulse rate was significantly increased 12 weeks after substituting brinzolamide compared with baseline with timolol ( ${ }^{*} \mathrm{p}<0.01$, Wilcoxon signed rank test).

second-line medication has been reported (Arici et al 1998; Shin 2000; Michaud and Friren 2001; O'Connor et al 2002; Martinez-de-la-Casa et al 2004; Shoji et al 2005; Tsukamoto et al 2005). Brinzolamide was reported to show an additional IOP reduction of $5.3 \mathrm{mmHg}$ (23.5\%) when added to latanoprost (Shoji et al 2005). It has also been reported that the addition of dorzolamide to latanoprost decreased IOP by $3.9 \mathrm{mmHg}(19.7 \%)$ compared with beta-blocker (2.5 mmHg; 12.3\%) (O’Conner et al 2002). Brinzolamide showed similar efficacy to timolol maleate when added to travoprost (Hollo et al 2006). In our study, IOP reductions from baseline at 4,8 , and 12 weeks after the substitution of brinzolamide $1 \%$ for timolol $0.5 \%$ were $0.8,1.2$, and $1.3 \mathrm{mmHg}$, respectively. The patients who enrolled in this study had already undergone treatment with latanoprost $0.005 \%$ and timolol $0.5 \%$. Nevertheless, the substitution of brinzolamide for timolol $0.5 \%$ further significantly decreased IOP. This result was similar to the IOP reduction observed by O'Conner et al (2002). The Early Manifest Glaucoma Trial Group reported that on follow-up, each $\mathrm{mmHg}$ reduction in IOP decreased the risk of disease progression by approximately $10 \%$ (Heijl et al 2002). Therefore, even though the reduction in IOP after the substitution

Table 2 Ocular discomfort

\begin{tabular}{ll}
\hline Blurring & 2 eyes $(10 \%)$ \\
Irritation (mild) & I eye $(5 \%)$ \\
Hyperemia (mild) & I eye $(5 \%)$ \\
\hline
\end{tabular}

Systemic adverse events were not observed. 
of brinzolamide for timolol was small ( $<2 \mathrm{mmHg}$ ), this IOP reduction could be considered clinically significant.

\section{Ocular discomfort}

The occurrence of ocular discomfort is related to the formula used for ophthalmic solutions. Brinzolamide is a white suspension. In our study, brinzolamide caused blurred vision ( 2 cases), and mild irritation and mild hyperemia (1 case) after switching from timolol. As with earlier studies, adverse events related to brinzolamide treatment generally occurred on instillation, were usually mild, resolved without treatment, and generally did not interrupt therapy continuation (Silver 1998; Barnebey and Kwok 2000; Martinez-de-la-Casa et al 2004) (ie, no patient was discontinued from the current study due to adverse events). It has also been reported that in comparisons of brinzolamide and timolol monotherapy (March and Ochsner 2000), brinzolamide produced fewer (3.3\%) ocular adverse events (burning/stinging) than timolol $(8.0 \%)$. The ocular discomfort of blurred vision was generally mild, and the medication was well tolerated. Therefore, evidence suggests that substituting brinzolamide for timolol may produce very few ocular adverse events in combination therapy with latanoprost.

\section{Systemic effects}

Non-selective and selective beta-blockers may have significant side effects on cardiovascular and respiratory organs, especially in elderly populations (Nelson et al 1986). When assessing a drug like timolol, which is used by a predominantly elderly population that is a priori at risk for cardiovascular and respiratory disease, it is often difficult to determine whether the suspect event is a consequence of the drug, the patients' age, an underlying medical condition, or a combination of factors. In agreement with a previous study, no clinically significant adverse effect on PR and BP occurred with brinzolamide (Silver 1998) and no systemic side effects were noted. Furthermore, increased PR is a potentially beneficial effect when substituting brinzomalmide for timolol. Topical beta-blockers can cause nocturnal hypotension in glaucoma patients (Heyreh et al 1999). Glaucoma is a disease that generally requires lifelong treatment. The most effective medications for long-term therapy are those that are efficacious, well tolerated, and show few side effects. In those patients in whom beta-blockers are contraindicated, brinzolamide offers an effective alternative.

\section{Conclusion}

The latanoprost/brinzolamide combination can have a significant additive effect on IOP reduction compared with the latanoprost/timolol combination. IOP reduction has been established to be an effective means of treating glaucoma (Mao et al 1991; Collaborative Normal-Tension Glaucoma Study Group 1998; AGIS Investigations 2000; Heijl et al 2002; Kass 2002; Tsukamoto et al 2005). To improve quality of life and vision, and to reduce the adverse events of glaucoma treatment, it is necessary to achieve a maximum IOP reduction with a minimum but adequate number of instillations. Substitution of brinzolamide $1 \%$ for timolol $0.5 \%$ in combination therapy with latanoprost $0.005 \%$ demonstrated superior and significant IOP reduction and improvement of PR. The ocular discomforts of blurred vision in 2 cases, mild irritation in 1 case, and mild hyperemia in 1 case were transient and mild after regimen change, and all resolved without treatment. The use of brinzolamide in place of timolol as a second-line medication in combination with latanoprost can be recommended for POAG or $\mathrm{OH}$ patients for better IOP control and fewer systemic side effects. However, this open non-randomized study evaluated a limited number of the patients treated in the short term, so that additional randomized studies on long-term administration and diurnal IOPs need to be considered.

\section{Disclosures}

None of the authors have any conflicts of interest to disclose.

\section{References}

AGIS Investigations. 2000. The Advanced Glaucoma Intervention Study (AGIS): The relationship between control of intraocular pressure and visual field deterioration. Am J Ophthalmol, 130:429-40.

Alm A, Widengard I, Kjellgren D, et al. 1995. Latanoprost administrated once daily caused a maintained reduction of intraocular pressure in glaucoma patients treated concomitantly with timolol. Br J Ophthalmol, 79:12-6.

Arici MK, Topalkana A, Guller C. 1998. Additive effect of latanoprost and dorzolamide in patients with elevated intraocular pressure. Int Ophthalmol, 22:37-42.

Barnebey H, Kwok SY. 2000. Patients' acceptance of switch from dorzolamide to brinzolamide for the treatment of glaucoma in a clinical practice setting. Clin Ther, 22:1204-12.

Brubaker RF, Ingnam CJ, Schoff EO, et al. 2000. Comparison of the efficacy of betaxolol -brinzolamide and timolol-dorzolamide as suppressors of aqueous humor flow in human subjects. Ophthalmology, 107:283-7.

Bucci MG. 1999. Italian Latanoprost Study Group. Intraocular pressurelowering effects of latanoprost monotherapy versus latanoprost or pilocarpine in combination with timolol: a randomized, observermasked multi-center study in patients with open-angle glaucoma. J Glaucoma, 8:24-30.

Camras CB, Alm A, Watson P, et al. 1996. Latanoprost, a prostgalndin analog, for glaucoma therapy. Efficacy and safety after 1 year of treatment in 198 patients. Latanoprost Study Groups. Ophthalmology, 103:1916-24.

Collaborative Normal-Tension Glaucoma Study Group. 1998. The effectiveness of intraocular pressure reduction in the treatment of normal-tension glaucoma. Am J Ophthalmol, 126:498-505. 
DeSantis L. 2000. Preclinical overview of brinzolamide. Surv Ophthalmol, 44:s119-29.

Heijl A, Laske MC, Bengtsson B, et al. 2002. Early Manifest Glaucoma Trial Group. Reduction of intraocular pressure and glaucoma progression: results from the Early Manifest Glaucoma Trial. Arch Ophthalmol, 120:2002.

Heyreh SS, Podhajsky P, Zimmerman MB. 1999. Beta-blocker eye drops and nocturnal arterial hypotension. Am J Ophthalmol, 128:301-9.

Higginbotham EJ, Feldman R, Stiles M, et al. 2002. Fixed Combination Investigative Group. Latanoprost and timolol combination therapy vs monotherapy: one-year randomized trial. Arch Ophthalmol, 120:915-22.

Holló G, Chiselita D, Petkova N, et al. 2006. The efficacy and safety of timolol maleate versus brinzolamide each given twice daily added to travoprost in patients with ocular hypertension or primary open-angle glaucoma. Eur J Ophthalmol, 16:816-23.

Kass MA, Heuer DK, Higginbotham EJ, et al. 2002. The Ocular Hypertension Treatment Study. A randomized trial determines that topical ocular hypotensive medication delays or prevents the onset of primary open-angle glaucoma. Arch Ophthalmol, 120:701-13.

Kobayashi H, Kobayashi K, Okinami S. 2004. Hypotensive effect and subjective symptoms after substitution of $1 \%$ dorzolamide by 1\% brinzolamide. Jpn J Clin Ophthalmol, 58:205-9.

Kobelt-Nguyen G, Gerdtham UG, Alm A, et al. 1998. Costs of treating primary open-angle glaucoma and ocular hypertension: a retrospective, observational chart review of newly diagnosed patients in Sweden and the United States. J Glaucoma, 7:95-104.

Kubota M, Hara T, Kubota S, et al. 2004. Ocular hypotensive effect of brinzolamide after switching from dorzolamide. Jpn JClin Ophthalmol, 58:301-3.

Larsson LI. 2001. Effect on intraocular pressure during 24 hours after repeated administration of the fixed combination of latanoprost $0.005 \%$ and timolol 0.5\% in patients with ocular hypertension. J Glaucoma, 10:109-14.

Mao LK, Stewart WC, Shields MB. 1991. Correlation between intraocular pressure control and progressive glaucomatous damage in primary open-angle glaucoma. Am J Ophthalmol, 111:51-5.

March WF, Ochsner LI. 2000. The Brinzolamide Long-Term Therapy Study Group. The long-term safety and efficacy of brinzolamide 1.0\% (Azopt) in patients with primary open-angle glaucoma or ocular hypertension. Am J Ophthalmol, 129:136-43.

Martinez-de-la-Casa JM, Garcia-Feijoo CJ, Mendez-Hernandez C, et al. 2004. Concomitant administration of travoprost and brinzolamide versus fixed latanoprost/timolol combined therapy. Three-month comparison of efficacy and safety. Curr Med Res Opin, 20:1333-9.

Michaud JE, Friren B. 2001. International Brinzolamide Adjunctive Study Group. Comparison of topical brinzolamide $1 \%$ and dorzolamide $2 \%$ eye drops given twice daily in addition to timolol $0.5 \%$ in patients with primary open-angle glaucoma or ocular hypertension. Am J Ophthalmol, $132: 235-43$
Nelson WL, Fraunfelder FT, Sills JM, et al. 1986. Adverse respiratory and cardiovascular events attributed to timolol ophthalmic solution, 1978-85. Am J Ophthalmol, 102:606-11.

O'Connor DJ, Martone JF, Mead A. 2002. Additive intraocular pressure lowering effect of various medications with latanoprost. Am J Ophthalmol, 133:836-7.

Sall K. 2000. The efficacy and safety of brinzolamide 1\% ophthalmic suspension (Azopt) as a primary therapy in patients with open-angle glaucoma or ocular hypertension. The Brinzolamide Primary Therapy Study Group. Surv Ophthalmol, 44:s155-62.

Schenker H, Maloney S, Liss C, et al. 1999. Patient preference, efficacy, and compliance with timolol maleate ophthalmic gel-forming solution versus timolol maleate ophthalmic solution in patients with ocular hypertension or open-angle glaucoma. Clin Therapeutics, 21:138-47.

Seong GJ, Lee SC, Lee JH, et al. 2001. Comparisons of intraocularpressure- lowering efficacy and side-effects of $2 \%$ dorzolamide and 1\% brinzolamide. Ophthalmologica, 215:188-91.

Shin D. 2000. Adjunctive therapy with brinzolamide 1\% ophthalmic suspension (Azopt) in patients with open-angle glaucoma or ocular hypertension maintained on timolol therapy. Surv Ophthalmol, 44:s163-8.

Shoji N, Ogata H, Suyama H, et al. 2005. Intraocular pressure lowering effect of brinzolamide $1.0 \%$ as adjunctive therapy to latanoprost $0.005 \%$ in patients with open angle glaucoma or ocular hypertension: an uncontrolled, open-label study. Curr Med Res Opin, 21:503-7.

Silver LH. 2000. Ocular comfort of brinzolamide 1.0\% ophthalmic suspension compared with dorzolamide $2.0 \%$ ophthalmic solution: Results from two multicenter comfort studies. The Brinzolamide Comfort Study Group. Surv Ophthalmol, 44:s141-5.

Silver LH. 1998. The Brinzolamide Primary Therapy Study Group. Clinical efficacy and safety of brinzolamide (Azopt), a new topical carbonic anhydrase inhibitor for primary open-angle glaucoma and ocular hypertension. Am J Ophthalmol, 126:400-8.

Stewart WC, Day DG, Stewart JA, et al. 2004. Short-term ocular tolerability of dorzolamide $2 \%$ and brinzolamide $1 \%$ versus placebo in primary open-angle glaucoma and ocular hypertension subjects. Eye, 18:905-10.

Tsukamoto H, Noma H, Matuyama S, et al. 2005. The efficacy and safety of topical brinzolamide and dorzolamide when added to the combination therapy of latanoprost and a beta-blocker in patients with glaucoma. J Ocul Pharmacol Ther, 21:170-3.

van der Valk R, Webers CAB, Schouten JSAG, et al. 2005. Intraocular pressure-lowering effects of all commonly used glaucoma drugs. A meta-analysis of randomized clinical trials. Ophthalmology, 112:1177-85 